\title{
Intervención para reducir la frecuencia de aparición de conductas disruptivas en un futbolista
}

\author{
Eugenio Antonio Pérez Córdoba \\ Universidad de Sevilla, España
}

\section{Rubén Salcedo Vázquez}

Universidad de Sevilla, España

\section{Omar Estrada Contreras}

Universidad CEU Cardenal Spínola, España

\section{Manuel Eugenio Huertas Castro}

Universidad de Sevilla, España

RESUMEN: El objetivo del presente estudio fue reducir en un futbolista senior la aparición de ciertas conductas disruptivas o consideradas hostiles ante situaciones de conflicto. Para ello, se ha implementado un diseño experimental A-B-A, observando en una primera fase (línea base) las conductas a modificar, interviniendo en la segunda mediante entrenamiento en técnicas de respiración y reforzamiento diferencial de conductas incompatibles y contemplando finalmente si los cambios experimentados se mantienen tras la retirada de la intervención. Los resultados revelan que la intervención ha producido cambios en el comportamiento del deportista, disminuyendo significativamente las conductas disruptivas y hostiles del mismo tales como protestas, insultos o amenazas, gestos de enfados y hostilidad o abandonar el campo. Se concluye que la intervención psicológica en el control de la respiración y el reforzamiento diferencial es eficaz para la mejora del autocontrol del deportista. De este modo, este gana un recurso para aumentar su rendimiento y, lo que, es más, la calidad de su relación con todo aquel que forme parte de su entorno deportivo.

PALABRAS CLAVES: conductas disruptivas, control emocional, futbol, psicología del deporte, diseño A-B-A

\section{Intervention to reduce the frequency of disruptive behaviors in a soccer player}

ABSTRACT: The objective of this study was to reduce the appearance of certain disruptive or hostile behaviors in conflict situations in a senior soccer player. For this purpose, an ABA experimental design was implemented, observing in the first phase (baseline) the behaviors to be modified, intervening in the second through training in breathing techniques and differential reinforcement of incompatible behaviors, and finally contemplating whether the changes observed are maintained after withdrawal of the intervention. The results revealed that the intervention produced changes in the athlete's behavior, significantly reducing disruptive and hostile behaviors. Research in this line continues to show improvements when working with psychological variables in athletes. In this case, the soccer player benefited from a psychological tool which he can turn to in order to manage his behavior in a more adapted way in situations of this kind. In this way, a resource is gained to increase his performance and, what is more, the quality of his relationship with everyone who is part of his sports environment is improved.

KEYWORDS: disruptive behaviors, emotional control, soccer, sports psychology, A-B-A design

Eugenio Antonio Pérez Córdoba es Psicólogo en la Universidad de Sevilla

Ruben Salcedo Vázquez es Psicólogo en la Universidad de Sevilla.

Omar Estrada Contreras es Psicólogo Universidad CEU San Pablo (Cardenal Spínola).

Manuel Eugenio Huertas Castro es Psicólogo en la Universidad de Sevilla.

La correspondencia de este artículo debe enviarse a: Eugenio Antonio Pérez Córdoba. Departamento de Psicología experimental. Universidad de Sevilla. E-mail: 


\section{Intervenção para reduzir a frequência do aparecimento de comportamentos disruptivos num jogador de futebol}

RESUMO: O objetivo deste estudo foi reduzir o aparecimento de certos comportamentos disruptivos ou hostis em situações de conflito num jogador de futebol sénior. Para isso, foi implementado um desenho experimental A-B-A, observando numa primeira fase (linha de base) os comportamentos a serem modificados, intervindo na segunda através de treino em técnicas de respiração e reforço diferencial de comportamentos incompatíveis, e finalmente contemplando se as mudanças vivenciadas são mantidas após a retirada da intervenção. Os resultados revelam que a intervenção produziu mudanças no comportamento do atleta, reduzindo significativamente os comportamentos disruptivos e hostis tais como protestos, insultos ou ameaças, gestos de protesto e hostilidade ou abandonar o campo. Conclui-se que a intervenção psicológica no controlo da respiração e o reforço diferencial são eficazes para a melhoria do autocontrolo do atleta. Dessa forma, o jogador ganha um recurso para aumentar o seu rendimento e, mais ainda, a qualidade do seu relacionamento com todos que fazem parte de seu ambiente desportivo.

PALAVRAS-CHAVE: comportamentos disruptivos, controlo emocional, futebol, psicologia do desporto, desenho A-B-A

Artículo recibido: 16/03/2021 | Artículo aceptado: 01/06/2021

En los últimos años se viene observando un incremento de los estudios volcados en el desarrollo e implementación de programas de intervención para mejorar los comportamientos éticos y adaptados de los deportistas (Cecchini et al., 2003). Actualmente se observan actitudes y conductas de violencia en las actividades y competiciones deportivas que se están produciendo de manera cotidiana (Demaría, 2004;

Gimeno, Sáenz et al., 2007; Weinberg, 2011). Además de actitudes y comportamientos que se saltan las normas y deportividad (Pelegrín, 2002, 2005; Gairín et al., 2014), así como agresiones de tipo verbal o física protagonizan un interesante campo de estudio e intervención para psicólogos/ as deportivos/as.

Pelegrín (2005) menciona la importancia de conocer qué origina o motiva que ciertos deportistas presenten menor autocontrol, actúen de un modo más impulsivo, tengan comportamientos desviados del juego limpio y reglado, denominándose hostiles o antideportivos (Dunn y Dunn, 1999; Pappas et al., 2004). Por ejemplo, tener una baja tolerancia (Anderson y Bushman, 2002); un patrón de comportamiento egocéntrico y competitivo, sumado a un bajo nivel de cooperación y preocupación por los demás (DeRosier et al., 1994); un débil manejo de las habilidades personales y sociales (Pepler et al., 1998) y finalmente, la exposición e imitación a modelos agresivos y violentos, predispone al deportista a un desajuste personal y social desde la infancia hasta la etapa adulta (Huesmann, 1998).

La impulsividad en las acciones del futbolista puede tener consecuencias negativas inmediatas en el rendimiento del propio equipo (amonestaciones o expulsiones, discusiones, clima negativo, etc.) (Navarro, et al., 1995), siendo importante trabajar con ellas

Se han realizado diversos estudios sobre la percepción de las conductas disruptivas sobre todo en escolares denotan- do discrepancias entre alumnos y profesores (Álvarez et al., 2016; Baños et al., 2018; Esteban et al., 2012).

Para poder intervenir con este tipo de conductas disruptivas, se han empleado diferentes técnicas, mayormente enfocadas a escolares (Banda y Sokolosky, 2012; Corsi et al., 2009; Fajardo-Vargas y Hernández-Guzmán, 2008; Gómez y Cuña, 2017; Graziano y Hart, 2016; Jodra et al., 2017; Leflot et al., 2010; Pérez et al., 2005; Ramírez, 2015; Ruiz et al., 2006; Sánchez-Rivas et al., 2015). Sin embargo, en los estudios anteriormente referenciados, la mayoría de las técnicas empleadas se orientan en el control de la activación y a la realización de conductas incompatibles (reforzamiento diferencial).

En un estudio de Amador et al. (2021) quitar naveira llevaron a cabo una intervención para la disminución de las conductas disruptivas en una escuela secundaria empleando el deporte como reforzamiento. Participaron 34 adolescentes de 13 años, emplearon un diseño tipo A B. Las técnicas de modificación de conducta que usaron fueron: contrato de contingencias, economía de fichas y costo de contingencias, obteniendo resultados positivos con disminución significativa de las conductas disruptivas de los participantes.

Uno de los objetivos de la preparación psicológica en el deportista es que obtenga autocontrol (Mujika et al. 2018; García-Naveira, 2010). De acuerdo con Arrieta y Chaverri (2019) el autocontrol es "el conjunto de procesos biológicos y cognitivos para inhibir, anular, o modificar una conducta, pensamiento o impulso indeseado" (p. 1013). Y es que, como se señala en estudios como los de Gil et al. (1993), Williams y Really (2000) o Gimeno, Buceta et al. (2007), el autocontrol se presupone como una de las variables psicológicas que convendría valorar para trabajar de cara a mejorar el rendimiento deportivo. En relación a esto último, García-Naveira (2017) realizó una investigación cuyo objetivo fue la aplicación de un programa de autocontrol a un entrenador de fútbol con 
17 años de experiencia, encontrando que el autocontrol fue efectivo para mejorar el rendimiento y la salud psicológica.

Así, el autocontrol, junto a otras variables como la atención, la motivación, la concentración, la autoconfianza, se relacionan de forma directa con la productividad deportiva (González et al., 2014; Pérez-Córdoba et al., 2020). De este modo y atendiendo a la pertinencia del campo de estudio que ofrece el autocontrol en el deporte, el objetivo general fue fomentar el autocontrol en un futbolista ante las decisiones arbitrales y el objetivo específico fue, disminuir las conductas disruptivas u hostiles o problemáticas emitidas por el deportista en dichas situaciones a través del entrenamiento psicológico en control de la respiración y el reforzamiento diferencial. La hipótesis que se estableció fue la siguiente: habrá una disminución de conductas disruptivas como efecto de la relajación y del reforzamiento diferencial de conductas incompatibles en el deportista.

\section{Método}

\section{Participantes}

El estudio constó de un solo participante $(\mathrm{N}=1)$, con una edad de 18 años, de un club de fútbol de un pueblo de la provincia de Sevilla (categoría "Primera Andaluza" siendo la sexta en orden desde la primera división), que accedió voluntariamente a participar en la investigación. En los partidos disputados en la temporada del estudio (20 partidos), fue amonestado con tarjeta amarilla en siete ocasiones, cuatro de ellas por protestas o gestos considerados como hostiles hacia el árbitro. Durante su trayectoria deportiva ha tenido bastantes episodios de respuestas de enfado u hostiles debidos a provocaciones, decisiones y acciones externas a él (hacia árbitros u otros futbolistas en mayor medida). El tiempo que dedica al entrenamiento durante la semana es de seis horas, a razón de una hora y media cada una de las cuatro sesiones de entrenamiento de cada semana.

Para registrar las conductas hubo dos observadores psicólogos pertenecientes al cuerpo técnico del equipo (trabajan en la cantera del club), con experiencia en registros observacionales y 5 años de labor profesional. Uno de ellos con especialidad en psicología deportiva fue el encargado de realizar la intervención con el jugador.

\section{Instrumentos}

Se diseñó una hoja de registro ad hoc para recoger las conductas a modificar del deportista, las situaciones que las provocan y las consecuencias que acarrean. Estas conductas disruptivas se encuentran sistematizadas y definidas en la Tabla 1.

También se usó una videocámara Sony HDR-CX240E Full HD, modelo HDRCX240EB.CEN que grabó las distintas sesiones. Para el trabajo individual en sala se utilizó un ordenador portátil, modelo HP Probook x360 11 G1 EE Intel Celeron N3350 de 4GB SSD128GB 11,6" táctil Grado B, un silbato (para simular los pitidos de los árbitros) y fotografías relacionadas con el caso, donde se veía a árbitros pitando y tomando decisiones sobre jugadas de fútbol.

\section{Procedimiento}

El trabajo fue demandado por el propio deportista, quien conocía a uno de los psicólogos que participaron en la investigación/intervención, de manera que, la participación fue voluntaria y a petición de él mismo, firmando, el jugador, un consentimiento informado, en el cual, se aseguró el respeto hacia su anonimato y confidencialidad.

Previo inicio de la intervención se realizó una entrevista inicial abierta, en la cual se analizó la situación del problema, definición, parámetros, determinantes, evolución y desarrollo de las conductas problemáticas. Se dedicó una semana previa al registro para el entrenamiento en observación y trabajo con hoja de registros con los observadores (comprobar la ausencia de alguna conducta inapropiada a observar, si hay algún problema a la hora de rellenar la hoja...).

Una vez finalizada la entrevista y el entrenamiento en observación, se llevó a cabo un diseño de reversión simple de tres fases o A-B-A (Barlow y Hersen, 1988) llevado a cabo a través de un registro observacional de medidas repetidas (estudio longitudinal), constituyendo así un estudio pre-experimental del tipo pre-test y post-test. Antes de comenzar la intervención, se llevó a cabo un periodo (cuatro sesiones de entrenamiento) de recogida de datos con el fin de que estos contribuyeran al cálculo del grado de acuerdo entre observadores. Para conocer el acuerdo entre los dos observadores, se consideraron los datos recogidos durante la fase piloto y los datos de la fase A del estudio. Utilizando el porcentaje de acuerdo de Bellack, como se hiciera en Cecchini et al (2003) para evaluar la fiabilidad en el criterio de acuerdo de estos, se obtuvo un acuerdo del $94.5 \%$.

Las conductas o respuestas a modificar, denominadas hostiles, antideportivas o problemáticas en este caso por su carácter perjudicial para el futbolista y su entorno deportivo (ver Tabla 1), fueron registradas en las distintas fases del diseño. Dichas respuestas problemáticas conforman la variable dependiente, que será definida y entendida como aque- 
llas acciones que se realicen tras la aparición no prevista de un estímulo externo y ajeno a la posibilidad de control del deportista. Estas conductas respondientes están caracterizadas por hostilidad, agresividad, recriminación, protesta o movimientos dirigidos o no a la fuente de dicha estimulación que supongan desafío, ataque o injusticia.

Por otro lado, los mencionados estímulos externos (ver Tabla 1), que actúan como elicitadores de las conductas inapropiadas, serán consideradas estímulos antecedentes en este caso, ya que ante su presencia aumenta la probabilidad de aparición del comportamiento inapropiado.

Las conductas problemáticas fueron observadas y anotadas en la hoja de registro, donde se reflejó su aparición (si aparece o no la conducta) ante la presencia de estímulos antecedentes o acciones elicitadoras, junto a las consecuencias que tras ellas se observaban de cara a detectar posibles factores y variables que intervienen en el mantenimiento del comportamiento en cuestión. Teniendo en cuenta que la duración de cada sesión de entrenamiento se acerca a las dos horas, se llevó a cabo un muestreo del tiempo que consistió en establecer dos periodos de observación: la primera media hora tras el calentamiento del equipo y la última, correspondiendo esta división respectivamente a la preparación física del entrenamiento y al "partidillo" final (simulando las competiciones).

Las técnicas que se emplearon en la parte de la intervención fueron las siguientes:

- Técnicas de respiración: como lo menciona Urra (2014), dentro de los procesos autonómicos, la respiración es el único que puede controlarse voluntariamente para disminuir el nivel basal de activación y generar respuestas de relajación que facilitan el equilibrio emocional. Por su parte Castellano (2011) destaca la respiración diafragmática para este tipo de casos.

Reforzamiento diferencial: como se indica en Olivares y Méndez (2011) esta técnica consiste en reforzar una o varias conductas diferentes de la conducta que se quiere reducir. Se espera pues que, al aumentar la frecuencia de esta nueva o nuevas conductas, disminuya progresivamente la frecuencia o desaparezca la conducta no deseada. Hay varias formas en las que se puede aplicar el reforzamiento diferencial: Reforzamiento diferencial de otras conductas (RDO), de conductas incompatibles (RDI) o de

\section{Tabla 1. Sistematización de categorías, descripción de las conductas inadaptadas a observar y de las acciones elicitadoras. Algunas de estas definiciones se apoyan en el trabajo de Navarro et al (1995)}

Estímulos antecedentes

Acciones, decisiones, estimulación externa, incontrolable e imprevista. El deportista estima estas acciones como injustas o indebidamente ejecutadas.
Variables dependientes

Conductas/Respuestas problemáticas, inadaptadas y hostiles. $\checkmark$ Tomar decisión arbitral. Por ejemplo: señalizar una acción del jugador como falta, fuera de juego, saque de banda, amonestarlo o expulsarlo.

$\checkmark$ Técnico hace corrección táctica o técnica, donde indica o lanza una consigna para mejorar algún aspecto técnico-táctico que el jugador está desarrollando. Por ejemplo: "No te pegues al central", "Fija la marca", "Orienta el control", "Mantén la zona".

$\checkmark$ Técnico exige más actitud, esfuerzo o algún factor que considera que no se está realizando todo lo bien que se podría. Por ejemplo: "Hay que trabajar más", "No esperes la pelota", "Hay que bajar más rápido".

$\checkmark$ Ser sustituido

$\checkmark$ Compañero de equipo recrimina alguna acción: un compañero se refiere al jugador mediante gestos o verbalmente para exigir o hacerle ver que no se está haciendo algo correctamente o que se está actuando de forma indebida. Por ejemplo: "Pero pásamela a mí", "Yo estaba solo", "No te quedes parado", "No conduzcas tanto".

$\checkmark$ Compañero se mofa o burla: compañero se dirige al jugador con afán de ridiculizarlo. Por ejemplo: "Centras como mi abuela", "Buen pase ese (irónicamente)".

$\checkmark$ Insulto o increpación directa.

$\checkmark$ Recibir falta: un jugador oponente comete una falta sobre él.
» Agresión física: entendida como todo contacto físico antirreglamentario del jugador con el cuerpo del contrario, en este caso producido como reacción o respuesta ante otra conducta no deseada. Por ejemplo: empujar, golpear, al contrario.

»Protesta:

Al árbitro: gestos o expresiones verbales dirigidos hacia el árbitro en protesta por una decisión tomada o por no haber adoptado una determinada decisión. Por ejemplo: el jugador le dice al árbitro "te estás equivocando con estas decisiones".

A compañeros o técnicos: el jugador se dirige al compañero o técnico con gestos o verbalmente, ante una acción que considera inadecuada, injusta o desacertada.

»Insultos 0 amenazas.

» Abandonar el ejercicio, entrenamiento o actividad que aún no ha acabado

»Muestras o gestos de enfado sin la intención de que sea percibida por otra persona, aunque en ocasiones esto no sea evitable. Por ejemplo: levantar los brazos en señal de desacuerdo, gritar, hacer un comentario del que se deduce enfado.

»Dirigirse de forma hostil hacia árbitro, compañero o técnico, usando un tono agresivo y amenazante Por ejemplo señalar al otro, levantarle la voz, acercarse al otro o incluso tocarle para que vea su enfado. 
tasas bajas (RDTB). En este caso se optó por utilizar el reforzamiento diferencial de conductas incompatibles. Para ello hay que identificar y seleccionar esta conducta incompatible que, en este caso, se eligió a mutuo acuerdo con el futbolista (con la que él se sentía más cómodo). Las conductas incompatibles en esta ocasión fueron, ante la presencia de estímulos o situaciones antecedentes, subirse las medias y posteriormente la realización de una o varias respiraciones (previamente entrenadas). Estas conductas se reforzaron halagando su capacidad de control y de mantener la calma (Ej.: "Bien aguantado $X$, antes no eras capaz de controlarte", "Gracias a callarte no te han sacado amarilla y eso favorece al equipo").

De cara a la delimitación y planificación de fases y sesiones (secuenciación, amplitud de fases e intervenciones propuestas para las mismas) se estableció la siguiente calendarización, considerando que se observaría y trabajaría con el deportista cuatro veces en semana:

Fase A (Línea base): tres semanas.

Periodo inicial de observación enfocado a la medición repetida de las conductas objeto de la investigación. La observación fue llevada a cabo por dos observadores mediante una hoja de registro y la grabación de las sesiones de entrenamiento, marcando esta la línea de comparación de las respuestas inadaptadas respecto a las siguientes fases. En total fueron doce las sesiones grabadas, a razón de cuatro por cada una de las semanas que duró esta fase.

Fase B: cuatro semanas.

De cara a reducir los comportamientos hostiles del futbolista ante las situaciones ya mencionadas, en primer lugar, se analizaron junto a este las repercusiones que tales comportamientos tenían para él y para el propio equipo (mal clima en el vestuario, consideración de deportista antideportivo y que no mira por el bien del equipo, malas relaciones con compañeros y técnicos...). Una vez abordada esta cuestión, por un lado, se trabajó el control de la activación (técnica de respiración), por otro, con una técnica operante para reducir y eliminar conductas, concretamente, el Reforzamiento Diferencial de Conductas Incompatibles (RDI). Durante las primeras sesiones se trabajó individualmente y en una sala apartada con el deportista, para después gradualmente ir introduciendo y trabajando estos aspectos en las situaciones de entrenamiento, sobre todo en los partidillos que recreaban los partidos de competición.

En esta fase, cuando el deportista realizó las conductas incompatibles fue reforzado por el entrenador y el resto de sus compañeros, con mensajes positivos, tales como: "Lo estás haciendo muy bien", "Estás mejorando mucho",
"Esta actitud ayuda mucho al equipo". Por otra parte, antes de finalizar cada sesión se comentaron los puntos trabajados, las dificultades, sensaciones de mejoría o lo que el deportista estimara oportuno.

En total el número de sesiones grabadas fue de 16, a razón de cuatro sesiones por cada una de las cuatro semanas que duro esta fase, como se he explicado anteriormente, dado que la duración de cada sesión de entrenamiento se acerca a las dos horas, se llevó a cabo un muestreo del tiempo que consistió en establecer dos periodos de observación: la primera media hora tras el calentamiento del equipo y la última, correspondiendo esta división respectivamente a la preparación física del entrenamiento y al "partidillo" final (simulando las competiciones).

\section{Vuelta a Fase A: tres semanas.}

Retirada de la intervención y observación para comprobar si descienden los cambios producidos durante la misma. Esta fase comprende la verdadera evaluación y mantenimiento de los cambios en el futbolista. Al comienzo de esta fase se habló con el jugador haciéndole ver que los jugadores del equipo al cual entrena estarían orgullosos de él y verían en su figura un modelo ejemplar. Se incidió en este punto, llegando a ser utilizado como elemento reforzador en estas situaciones. El tiempo y número de sesiones fue el mismo que en la Fase $\mathrm{A}$.

\section{Análisis estadístico}

Para determinar la tasa de cambio que experimentó la conducta del futbolista se utilizó la técnica conocida como "Split middle" (Crosbie, 1987), en la cual se ubican las medianas de los datos en la primera y segunda mitad de la línea base, dibujando una línea. Esta línea se extiende hasta la fase de intervención y se cuentan los puntos que están por encima o debajo de la misma (Matyas y Greenwod, 2014). No obstante, para confirmar un cambio significativo del comportamiento de los datos, o lo que es lo mismo, para rechazar la hipótesis de nulidad, se aplicó la prueba binominal.

\section{Resultados}

En primer lugar, como se puede apreciar en la Tabla 2, hay un claro descenso de las conductas problemáticas desde que la intervención comienza a llevarse a cabo. Si atendemos a los datos generales totales registrados en cada fase, podemos observar como desde la fase de línea base (A) a la fase de retirada de la misma $\left(A^{\prime}\right)$, las respuestas antidepor- 
tivas han experimentado un cambio notable (Media $\mathrm{A}=6.25$; Media B=1.56; Media Á=.37).

En la Tabla 2, también se reflejan las respuestas específicas observadas en la primera y última fase del diseño experimental, fases pre y post intervención, observándose también el descenso de las conductas problema. A su vez, podemos ver como las respuestas específicas de "Protesta", "Gestos de enfado" y "Dirigirse de forma hostil" son las que se dan con más frecuencia, por lo que más adelante nos centraremos en un análisis exclusivo de estas y su cambio.

Tabla 2. Frecuencia total y medias por sesión de conductas disruptivas generales durante la fase $A$ de línea base, en la $B$ en período de intervención y en la $\mathrm{A}^{\prime}$ tras la retirada de la intervención $(n=1)$

\begin{tabular}{cccc}
\hline & Fase $A$ & Fase B & Fase $A^{\prime}$ \\
\hline $\begin{array}{c}\text { Conductas } \\
\text { Disruptivas } \\
\text { Generales }\end{array}$ & $75(\mathrm{M}=6.25)$ & $25(\mathrm{M}=1.56)$ & $6(\mathrm{M}=.5)$ \\
\hline Protesta & $36(\mathrm{M}=3)$ & $12(\mathrm{M}=.75)$ & $3(\mathrm{M}=.25)$ \\
\hline $\begin{array}{c}\text { Insulto/Ame- } \\
\text { naza }\end{array}$ & $2(\mathrm{M}=.16)$ & $1(\mathrm{M}=.06)$ & $1(\mathrm{M}=.08)$ \\
\hline $\begin{array}{c}\text { Abandonar } \\
\text { Gestos Enfado }\end{array}$ & $20(\mathrm{M}=.08)$ & 0 & 0 \\
\hline Hostilidad & $16(\mathrm{M}=1.66)$ & $6(\mathrm{M}=.37)$ & $2(\mathrm{M}=.16)$ \\
\hline
\end{tabular}

Para determinar la tasa de cambio que experimentó la conducta del futbolista se utilizó la técnica conocida como "Split middle" (Crosbie, 1987). En las figuras 1, 2, 3 y 4, podemos apreciar como la tendencia de los datos en este di-

Figura 1. Repuestas problemáticas totales en las distintas sesiones de la fase $A$. También se señala el punto mediano que establece el criterio para la pendiente de los datos según la técnica split middle

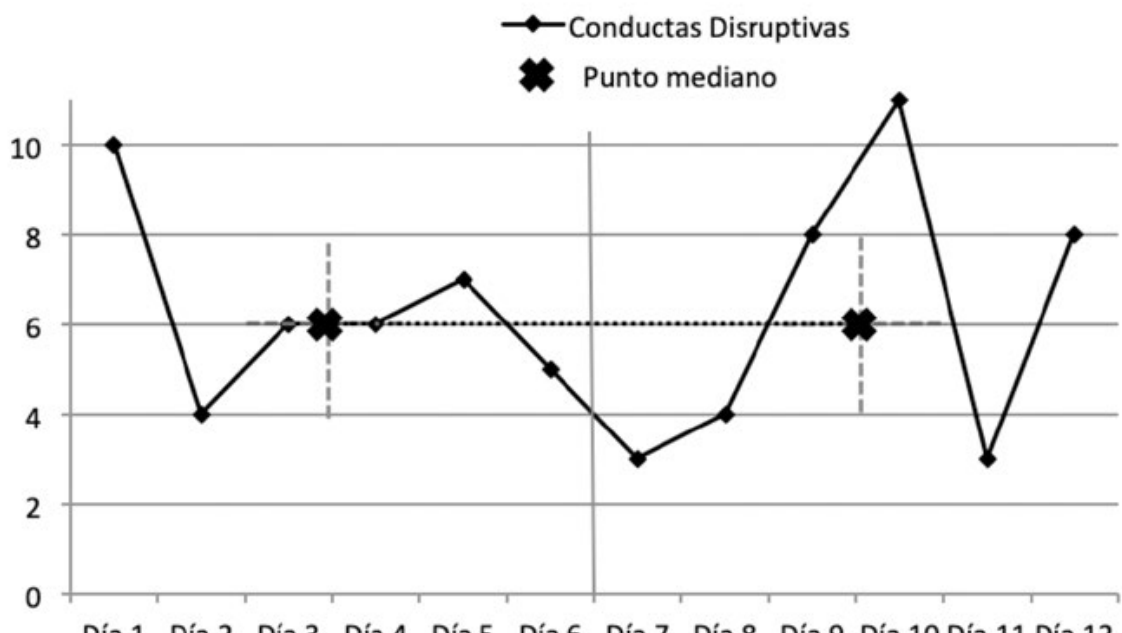

seño experimental apunta a que estos, tras la aplicación de la intervención propuesta, descienden de manera evidente y marcada. En dichas gráficas, mostradas a continuación, se refleja la frecuencia de estas conductas en cada una de las sesiones y, por consiguiente, su evolución a lo largo de las fases. En las figuras 1 y 2 de presentan de forma general y en el resto de figuras se realiza un análisis de las conductas específicas.

Figura 2. Repuestas problemáticas totales en las distintas sesiones de la fase A'. También se señala la prolongación de la línea que marca la pendiente de los datos de la Fase A o "línea de split middle"

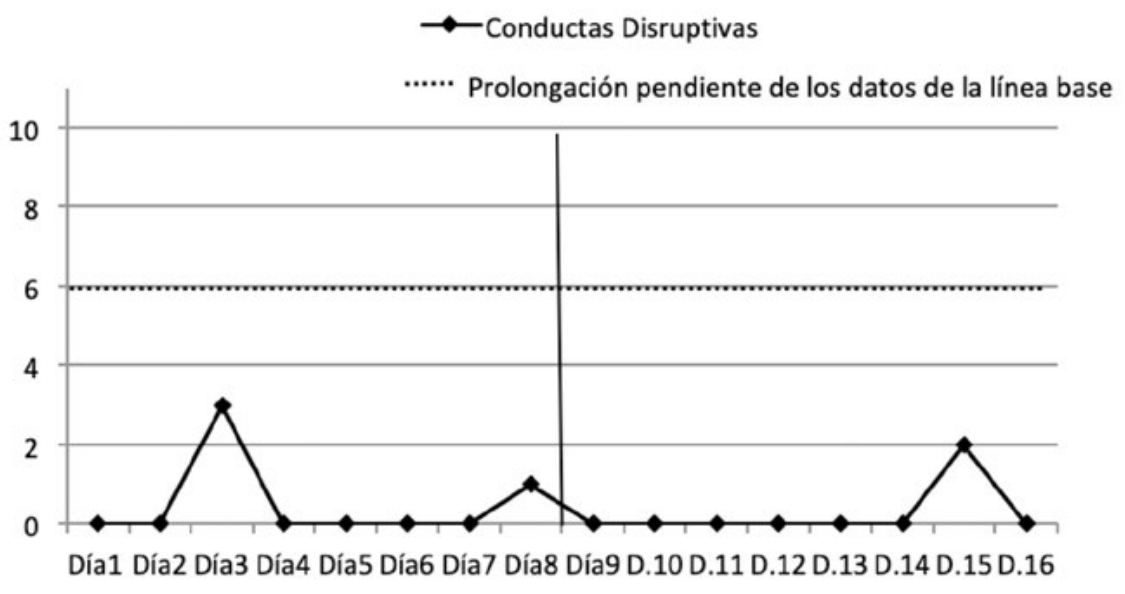

Si se observa la Figura 2, ningún dato supera la línea split middle original, lo que ya nos revela que el cambio experimentado por los datos sigue un patrón descendente, cosa que ocurrirá en todas las conductas observadas, como vemos aquí y veremos seguidamente.

De este modo, obtenemos una $p<.001$, por lo que rechazamos la hipótesis nula y, por consiguiente, una posible explicación de la reducción de respuestas problemáticas debida al azar.

A continuación, se realizará el mismo proceso de análisis con las conductas específicas que más incidencia mostraron, siendo estas la de protesta, los gestos de enfado y dirigirse de forma hostil a técnicos, árbitros o compañeros. Los datos mostraron una disminución en la fase posterior a la de la llevada a cabo de la intervención (Fase A'). No obstante, parece pertinente analizarlas por separado para observar posibles diferencias entre estas.

Como podemos ver en las Figuras 3 y 4, como ya aconteciera en las Figuras 1 y 2, no se observó ningún resultado por encima de la línea original split middle. Obteniendo de nuevo una $\mathrm{p}<.001$, podríamos afirmar que la alteración de los datos no se debe al azar, por tanto, rechazamos la hipótesis nula.

En lo que respecta a las conductas de gestos de enfado, y pese a observar también como los datos han disminuido 
Figura 3. Repuestas de protesta en las distintas sesiones de la fase $A$

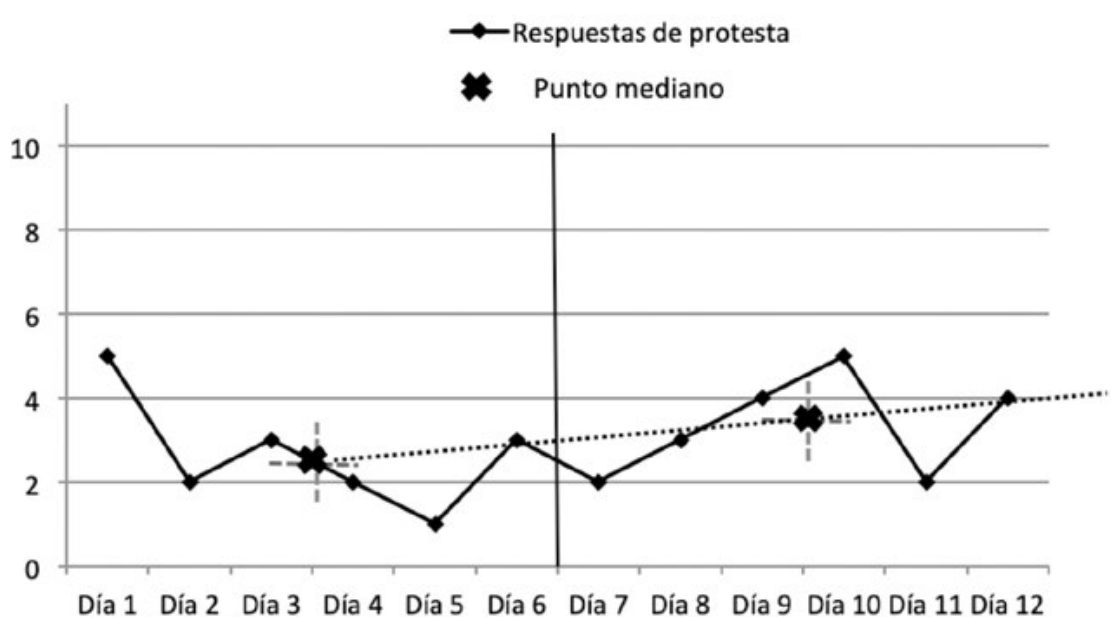

Figura 4. Repuestas de protesta en las distintas sesiones de la fase A'. Prolongación de la "línea de split middle".

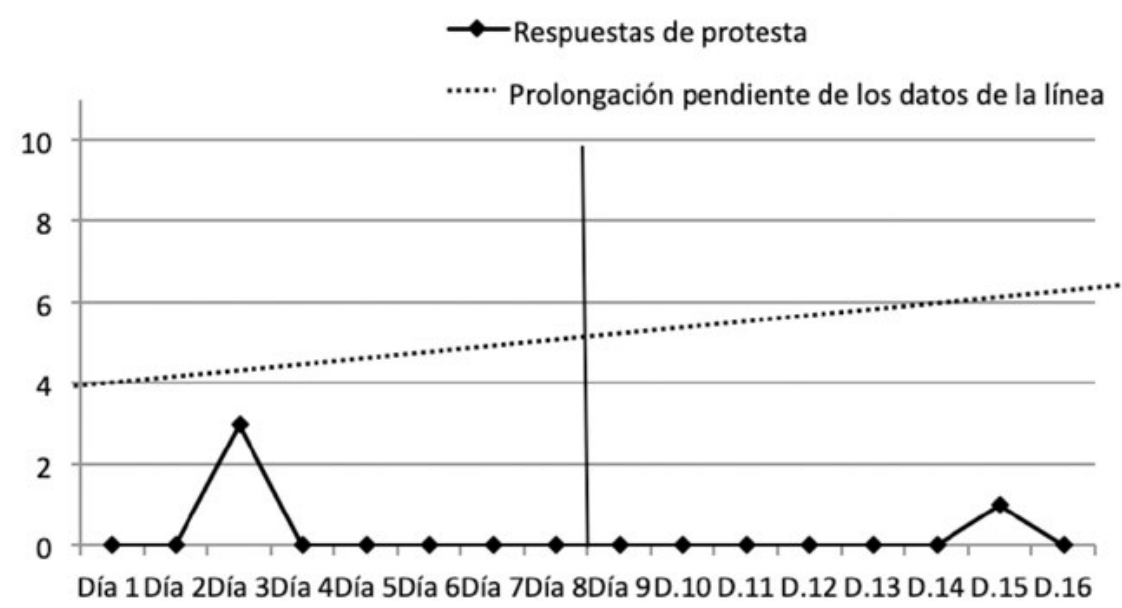

notablemente (Tabla 2), podemos ver como dos conductas no estuvieron por debajo de la línea split middle, cosa que no ocurrió en otras conductas. No obstante, con una $p<.001$, seguiríamos rechazando la nulidad de los datos y la influencia del azar como explicador de los cambios presenciados.

Finalmente, atendiendo ahora a las respuestas de "dirigirse con hostilidad a técnicos, árbitros o compañeros", la Tabla 2 y el análisis de los datos, con una $\mathrm{p}<.001$, vuelven a evidenciar un cambio de estos no debido al azar, rechazándose así la hipótesis nula.

\section{Discusión}

En el deporte de competición, uno de los objetivos primordiales es conseguir que los deportistas rindan al máximo nivel posible. De este modo, sus probabilidades de alcanzar logros deportivos como fichar por un equipo mejor, ganar un campeonato o jugar de titular aumentarían (Gimeno, Sáenz et al., 2007). Como se comentó anteriormente, en la consecución de un rendimiento óptimo las variables psicológicas juegan un papel crucial, considerando el autocontrol o el presentar un comportamiento adaptado a los requisitos de la competición entre estas. En esta línea, como afirma Pelegrín (2005), las conductas agresivas, inadaptadas, irrespetuosas, hostiles y alejadas de la norma requieren de una intervención para mejorar la deportividad y el control de los deportistas.

En el presente estudio, se intervino con un futbolista hacia la modificación y adquisición de comportamientos que le permitan aprender a controlar la expresión de la ira para contrarrestar la excitación y la tensión en situaciones conflictivas. Los resultados indican que la intervención llevada a cabo con el fin de reducir los comportamientos problemáticos y hostiles del futbolista fue eficaz, es decir, se aceptó la hipótesis de trabajo, ya que hubo una disminución de conductas disruptivas debido al efecto del reforzamiento diferencial de conductas incompatibles en el futbolista participante. Esta intervención tuvo resultados similares en la disminución de conductas disruptivas en los estudios con técnicas conductuales realizados por Banda y Sokolosky (2012); Corsi et al. (2009); Graziano y Hart (2016); Leflot et al. (2010); Pérez et al. (2005); Ruiz et al. (2006). Coincidimos también con los resultados encontrados por Amador et al. (2021) que obtuvieron disminución de conductas disruptivas en alumnos de secundaria (diseño $N=1$ ), aunque no emplearon las mismas técnicas, pero el reforzamiento de conductas distintas a la línea base fue efectivo. La diferencia que podemos marcar, es que no usaron otra condición de evaluación posterior al tratamiento, para medir la estabilidad de los cambios. Además, se consiguió que el deportista participante obtuviera un autocontrol, siendo similar al resultado conseguido por García-Naveira (2017) con un entrenador de fútbol, pero diferenciando en el tipo de evaluación de línea base, ya que empleo cuestionarios y autoinformes. El tipo de técnicas que coincidieron fueron la de relajación, pero, las de modificación de conducta fueron autorregistros que llevaba a cabo el propio entrenador y algunas de tipo cognitivo. Hubo también una diferencia en cuanto al lugar de administración de la intervención, ya que se llevó a cabo en un despacho y en este estudio fue en el campo de fútbol. Estos cambios significativos observados en las respuestas del deportista, nos dicen que, para él, las técnicas y estrategias entrenadas se presentan como una herramienta de la que puede hacer uso en aquellas ocasiones en las que lo precise, como un recurso futbolístico y psicológico más. Como observamos, el análisis de los resultados refleja dichas alteraciones significativas de la conducta en el sentido esperado en respuestas como la protesta, gestos de enfado y dirigirse con hostilidad 
hacia otros. En lo que concierne a conductas como la agresión física, insulto/amenaza o abandonar, al no observarse apenas incidencia de estas en la línea base, no se procedió a un análisis más detallado como si se hiciera en el resto de los casos. Pese a ello, se puede destacar que estas conductas no existieron prácticamente en la vuelta a línea base.

Hay que de resaltar el hecho de que, al tratarse de un estudio de caso único, no podemos afirmar que los citados cambios experimentados son extrapolables a cualquier otra población con dificultades similares, lo que invita a pensar que son necesarios nuevos estudios de casos individuales y colectivos. No obstante, con este futbolista, la intervención combinando técnicas de respiración y reforzamiento diferencial de conductas incompatibles sí tuvo un efecto importante en su autocontrol y una mejor gestión de situaciones que antes, en la mayoría de los casos, promovían con alta probabilidad respuestas antideportivas, inadaptadas y contraproducentes para él y su equipo.

Esta línea de trabajo también podría tener cabida a nivel de deportistas más jóvenes. Últimamente se observa un incremento en la incidencia de comportamientos agresivos y antideportivos a cortas edades (Borrás et al., 2001; Pelegrín, 2002, 2005), que da lugar a una mayor cotidianidad de actitudes intolerantes y de situaciones de violencia en la que jóvenes deportistas participan (Weinberg, 2011; Gairín et al., 2014). Implantar desde pequeños valores y modelos de comportamiento deportivos (jugadores capaces de controlarse y de comportarse deportivamente, en lugar de ser conflictivos, violentos e impulsivos) supondría un avance de cara a prevenir casos problemáticos como el que nos atiende en este estudio.

Para concluir, cabría resaltar que como en otros ámbitos de intervención a nivel psicológico, la predisposición y compromiso colaborativos son importantes para que lo realizado sea útil. Así, si el futbolista en este caso no quiere cambiar su actitud o conducta y se respalda en afirmaciones del tipo "yo soy así", el cambio es más difícil (González et al., 2014). Esto no sucede en este caso, ya que el jugador si proyecta su voluntad de cambio desde un primer momento, haciendo posible y más fácil la modificación conductual a través de las herramientas y estrategias ofrecidas y trabajadas.

Finalmente, de cara a futuras investigaciones e intervenciones, sería interesante aumentar el tiempo de intervención, así como un seguimiento más prolongado, además de trabajar otras conductas específicas, así como complementar las técnicas utilizadas con otras de reestructuración cognitiva si el caso lo requiriera, de habilidades sociales, u otras posibilidades para trabajar también autocontrol en situaciones de ira o conflictividad.

\section{Referencias}

Anderson, C. A. y Bushman, B. J. (2002). Human Agression. Annual Review of Pshycology, 53, 27-51. https://doi.org/10.1146/ annurev.psych.53.100901.135231

Álvarez, M., Castro, P., González-González de Mesa, C., Álvarez, E. y Campo, M.A. (2016). Conductas disruptivas desde la óptica del docente: validación de una escala. Anales de Psicología, 32(3), 855-862. https://doi.org/10.6018/analesps.32.3.223251

Amador-Salinas, J. G., González Rivera, V. y Pérez Martínez, G. (2021). Disminución de conductas disruptivas en clases usando técnicas conductuales, deporte y motivación. Revista Digital Internacional De Psicología y Ciencia Social, 7(1), 68-85. https:Il doi.org/10.22402/i.rdipycs.unam.7.1.2021.332.68-85

Arrieta, L. A. y Chaverri, P. (2019). Componentes del autocontrol. Revista Electrónica de Psicología Iztacala, 22(5), 1005-1023.

Banda, D. R. y Sokolosky, S. (2012). Effectiveness of noncontingent attention to decrease attention-maintained disruptive behaviors in the general education classroom. Child and Family Behavior Therapy, 34(2), 130-140. https://doi.org/10.1080/0731 7107.2012 .684646

Baños, R., Ortiz-Camacho, M., Baena-Extremera, A. y Zamarripa, J. (2018). Efecto del género docente en la importancia de la educación física, clima motivacional, comportamientos disruptivos, la intención de la práctica futura y rendimiento académico. Retos, 33, 252-257. https://doi.org/10.47197/retos. v0i33.59991

Barlow, D. H. y Hersen, M. (1988). Diseños experimentales de caso único. Martínez Roca.

Borrás, A., Palou, P. y Ponseti, F. X. (2001). Evaluación del fairplay y conductas violentas durante la práctica deportiva de estudiantes de magisterio en educación física. En J.F. Campos, S. Llanas y R. Aranda (Eds.), Nuevas aportaciones al estudio de la actividad física y el deporte (pp. 86-92). II Congreso de Ciencias de la Actividad Física y el Deporte. Marí Montañana.

Corsi, E., Barrera, P., Flores, C., Perivancich, X. y Guerra, C. (2009). Efectos de un programa combinado de técnicas de modificación de conductual para la disminución de la conducta disruptiva y el aumento de la conducta prosocial en escolares chilenos. Acta Colombiana de Psicología, 12(1), 67-76.

Castellano, M. (2011). La respiración consciente como factor principal de la relajación en la educación física escolar. EmásF Revista Digital de Educación Física, 3(13), 19-31.

Cecchini, J. A., Montero, J. y Peña, J. V. (2003). Repercusiones del Programa de Intervención para Desarrollar la Responsabilidad Personal y Social de Hellison sobre los comportamientos de fair-play y el autocontrol. Psicothema, 15(4), 631-637.

Crosbie, J. (1987). The stability of the binomial test to control Type I error with single-subject data. Behavioral Assessment, 9, 141150.

Demaría, M. J. (2004). Una propuesta para la prevención de la agresión desde el área de educación física. Revista Digital EFDeportes.com, 76.

DeRosier, M. E., Cillessen, A. H. N., Coie, J. D. y Dodge, K. A., (1994). Group social context and children's aggressive behavior. Child Development, 65(4), 1068-1079. https://doi. org/10.2307/1131305 
Dunn, J. G. H. y Dunn, J. C. (1999). Goal orientations, perceptions of aggression, and sports personship in elite male youth ice hockey players. Sport Psychologist, 13(2), 183-200.

Esteban, R., Fernández, J. G., Díaz, A. y Contreras, O. R. (2012). Las conductas que alteran la convivencia en las clases de educación física. Revista Internacional de Medicina y Ciencias de la Actividad Física y el Deporte, 12(47), 459-472.

Fajardo-Vargas, V. y Hernández-Guzmán, L. (2008). Tratamiento Cognitivo-Conductual de la conducta agresiva infantil. Revista Mexicana de Análisis de la Conducta, 34(2), 369-387. https://doi. org/10.5514/rmac.v34.i2.16216

Gairín, J., Muñoz, J. L., Castro, D. y Díaz-Vicario, A. (2014). Causas de la intolerancia en las competiciones deportivas escolares: elaboración de un código de conducta. Revista de Psicología del Deporte, 23(2), 255-265.

García-Naveira, A. (2010). El psicólogo del deporte en el alto rendimiento: aportaciones y retos futuros. Papeles del Psicólogo, 37(3), 259-268.

García-Naveira, A. (2017). Entrenamiento psicológico para la mejora del autocontrol en un entrenador de fútbol [Psychological training for improving self-control in a football coach]. Acción Psicológica, 14(1), 27-42. https://doi.org/10.5944/ ap. 14.1.79252

Gil, J., Capafons, A. y Labrador, F. (1993). Variables físicas y psicológicas predictoras del rendimiento deportivo y del cambio terapéutico. Psicothema, 5(1), 97-110.

Gimeno, F., Buceta, J. M. y Pérez-Llantada, M. C. (2007). Influencia de las variables psicológicas en el deporte de competición: Evaluación mediante el cuestionario características psicológicas relacionadas con el rendimiento deportivo. Psicothema, 19(4), 667-672.

Gimeno, F., Sáenz, A., Ariño, J. V. y Aznar, M. (2007). Deportividad y violencia en el fútbol base: un programa de evaluación y de prevención de partidos de riesgo. Revista de Psicología del Deporte, 16(1), 103-118.

González, G., Del Castillo, O. y Romero, S. (2014). Control analysis of the attitude in a team of football players. Journal of Sport and Health Research. 6(3), 241-252.

Gómez, M. C. y Cuña, A. R. (2017). Estrategias de intervención en conductas disruptivas. Educação por Escrito, 8(2), 278-293. https://doi.org/10.15448/2179-8435.2017.2.27976

Graziano, P A. y Hart, K. (2016). Beyond behavior modification: Benefits of social/emotional self-regulation training for preschoolers with behavior problems. Journal of School Psychology, 58, 91-111. https://doi.org/10.1016/j.jsp.2016.07.004

Huesmann, L. R. (1998). La conexión entre la violencia en el cine y la televisión y la violencia real. En J. Sanmartín, J. S. Grisolía y S. Grisolía (Eds.), Violencia, televisión y cine (pp. 87-132). Ariel.

Jodra, P., Domínguez, R. y Maté-Múñoz, J. L. (2017). Incidencia de la práctica deportiva en la conducta disruptiva de niños y adolescentes. Ágora para la Educación Física y el Deporte, 19(23), 193-206.

Leflot, G., Van-Lier, P. A. C., Onghena, P. y Colpin, H. (2010). The role of teacher behavior management in the development of disruptive behaviors: An intervention study with the Good Behavior Game. Journal of Abnormal Child Psychology, 38, 869-882. https://doi.org/10.1007/s10802-010-9417-4
Matyas, T. A. y Greenwod, K. M. (2014). Serial Dependency in single case time series. En Franklin, R.D., Allison, D.B., \& Gorman, B.S. (Eds). Design and Analysis of Single-Case Research (pp. 215244). Psychology Press.

Mujika, I., Halson, S., Burke, L. M., Balagué, G. y Farrow, D. (2018). An integral multifactorial approach to periodization for optimal performance in individual and team sports. International journal of Sports Physiology and Performance, 13(5), 538-561. https://doi.org/10.1123/ijspp.2018-0093

Navarro, J. I., Amar. J. R. y González, C. (1995). Ansiedad pre-competitiva y conductas de autocontrol en jugadores de fútbol. Revista de Psicología del Deporte, 4(2), 7-17.

Olivares, J. y Méndez, F. X. (2011). Técnicas de modificación de conducta. Pirámide.

Pappas, N. T., Mckenri, P. C. y Catlett, B. S. (2004). Athlete aggression on the rink and off the ice: Athlete violence and aggression in hockey and interpersonal relationships. Men and Masculinities, 6(3), 291-312. https://doi.org/10.1177/1097184X03257433

Pelegrín, A. (2002). Conducta agresiva y deporte. Cuadernos de Psicología del Deporte, 2(1), 39-56.

Pelegrín, A. (2005). Detección y valoración de la incidencia de las actitudes antideportivas durante la competición. Cuadernos de psicología del Deporte, 5(1-2), 133-142.

Pepler, D., Craig, M. y Roberts, W. L. (1998). Observation of aggressive and nonaggressive children on the school playground. Merrill-Palmer Quarterly, 44(1), 55-76.

Pérez, V., Fernández, A. M., Rodríguez, J. y De la Barra, F. (2005). Evaluación del efecto de una intervención conductual en la agresividad escolar. Terapia Psicológica, 23(1), 91-98.

Pérez-Córdoba, E., Estrada, O., Gutiérrez, M. T y Ramírez, O. (2020). Nivel de Activación Óptimo y Rendimiento en un jugador de Fútbol no Profesional. Revista de Psicología Aplicada al Deporte y al Ejercicio Físico, 5(1), Artículo e5. https://doi.org/10.5093/ rpadef2020a7

Ramírez, M. (2015). Tratamiento cognitivo-conductual de conductas disruptivas en un niño con TDAH y trastorno negativista desafiante. Revista de Psicología Clínica con Niños y Adolescentes, 2(1), 45-54.

Ruiz, M. R., Pino, M. J. y Herruzo, J. (2006). Revisión de la técnica "el juego del buen comportamiento". Análisis y Modificación de Conducta, 32(144), 553-574.

Sánchez-Rivas, E., Sánchez-Rodríguez, J. y Ruiz-Palmero, J. (2015). Método para el tratamiento educativo de las conductas disruptivas en el aprendizaje deportivo. Revista Iberoamericana de Psicología del Ejercicio y el Deporte, 10(2), 225, 234.

Urra, B. (2014). Evaluación de la efectividad del entrenamiento de estrategias de afrontamiento en el nivel de ansiedad precompetitiva en tenimesistas. Revista de Psicología del Deporte, 23(1), 67-74.

Weinberg, R. (2011). Improving sportsmanship in youth sports: How research informs practice. En Libro de resúmenes del II Seminario Nacional Fomento de la Deportividad y Prevención de la Violencia en el Deporte en Edad Escolar (pp. 7-18). Vitoria-Gasteiz.

Williams, A. y Reilly, T. (2000). Talent identification and development in soccer. Journal of Sports Sciences, 8, $657-667$. https:// doi.org/10.1080/02640410050120041 